\title{
FROM PAHS TO SOLID CARBON
}

\author{
C. Jäger ${ }^{1}$, H. Mutschke ${ }^{2}$, T. Henning ${ }^{3}$ and F. Huisken ${ }^{1}$
}

\begin{abstract}
Carbonaceous grains represent a major component of cosmic dust. The review will summarize new results in laboratory investigations of carbonaceous dust components. The nanometer-sized carbon particles are supposed to represent a blend of differently structured carbon including graphitic, diamond-like, fullerene-like and chain-like components on a subnanometer or nanometer scale. Recent models used to explain the structure of gas-phase condensed carbon nanoparticles are discussed. Possible formation pathways of carbonaceous grains from molecular components and clusters and the role of polycyclic aromatic hydrocarbons (PAHs) and fullerenes are disclosed.
\end{abstract}

\section{Introduction}

Carbonaceous grains belong to the most abundant cosmic dust components. Cosmic dust absorbs and scatters stellar light and radiates the energy in the IR and millimeter range. Very small dust grains influence the heating rate of gas in the interstellar medium (ISM) by the photoelectric effect. In addition, surfaces of dust grains are efficient factories for the formation of molecular hydrogen and larger organic molecules. In the diffuse ISM, grain shattering and sputtering processes can erode and fragment grains into smaller units and therefore grains can act as precursors of organic molecules. Carbon and hydrogen atoms constitute the basis of a rich organic chemistry in the solar system and in the interstellar medium and finally for life on earth and, perhaps, on other planets. However, the problem with the carbon dust component is the huge diversity of allotropes and structures that can be mixed up in one grain. Due to the processing of the dust grains in the interstellar medium, the composition and structure is constantly modified. Large

\footnotetext{
1 Max-Planck-Institute for Astronomy, Heidelberg and Institute of Solid State Physics, Friedrich Schiller University, Helmholtzweg 3, 07743 Jena, Germany

2 Astrophysical Institute and University Observatory, Friedrich Schiller University, Schillergässchen 2-3, 07745 Jena, Germany

3 Max-Planck-Institute for Astronomy, Königstuhl 17, 69117 Heidelberg, Germany
} 
PAH molecules are involved in these processes both as precursors of condensation and products of grain disintegration. Consequently, the spectral properties of cosmic carbon grains, which are strongly related to the structure and composition of the carbonaceous materials, are changing considerably from circumstellar to interstellar to protoplanetary environments. In order to "understand" the spectral signature of the cosmic carbon component, one has to study the spectral properties of differently structured carbon materials in the laboratory.

\section{Solid forms of carbon}

For carbon, the possibilities to form solid structures are extremely manifold because of its ability to occur in different hybridization states. Carbon exist in different allotropes including diamond, graphite, polyynes and fullerenes. Further solid forms of carbon are graphene and nanotubes, new forms of solid carbon, which are based on graphitic or fullerene structures.

In the diamond lattice, the tetrahedrally coordinated carbon atoms are covalently bound to each other via four strong $\sigma$ bonds. Consequently, diamond is an insulator having a large band gap of $5.5 \mathrm{eV}$. In graphite, the three $\mathrm{sp}^{2}$ hybrid orbitals have a trigonal planar symmetry and can form $\sigma$ bonds with the adjacent carbon atoms whereas the nonhybridized p electron is able to form an additional $\pi$ bond. This results in infinite layers of condensed benzene rings with delocalized $\pi$ electrons between them. Therefore, graphite represents a zero band gap material.

In polyynes, the carbon atoms are sp hybridized forming two sp hybrid orbitals with two remaining p orbitals. The sp hybridized carbon atoms build up chains with alternating triple bonds. Polyyne molecules or radicals are found to exist in the ISM. These chain-like structures form in gas-phase condensation experiments and they are supposed to be precursors of fullerenes or fullerene-like carbon grains (Ravagnan et al. 2002).

Apart from the distinct hybridization states, there exist mixed hybridization states which build up curved structures. In the perfect form, they are closedshell molecules consisting of only carbon, called fullerenes (the fourth allotrope of carbon). They can be produced in gas-phase condensation reactions such as by laser ablation or arc discharge of carbon electrodes in helium gas atmosphere (Kroto et al. 1985; Krätschmer et al. 1990). The curvature is achieved by the incorporation of pentagons and heptagons. The deviation of the planar structure results in a higher s-character of the $\pi$ orbital and a mixed hybridization state (Haddon 1993). $\mathrm{C}_{60}, \mathrm{C}_{70}$, or smaller fullerenes are not "superaromatic" as they tend to avoid double bonds in the pentagonal rings. $\mathrm{C}_{60}$ behaves like an electron deficient alkene and reacts readily with electron-rich species. Many fullerenes have now been detected in carbon soot, uncovered by electron microscopy, including very small ones such as $\mathrm{C}_{20}$ or $\mathrm{C}_{34}$.

Carbon nanotubes (CNTs), the fifth form of solid carbon, can exist as singlewalled or multi-walled species with several nested cylinders. Multi-walled forms can be produced in gas-phase condensations together with other particulate carbon 
forms (Iijima 1991). Single walled CNTs up to lengths of about $1 \mu \mathrm{m}$ can be produced using catalysts (Harris 2009). A single-wall CNT can be imagined as a graphene sheet rolled at a certain chiral angle. CNTs can grow up to lengths over $1 \mu \mathrm{m}$ and with a range of diameters from $1 \mathrm{~nm}$ (for single walled) to around $50 \mathrm{~nm}$ for multi walled ones. The electronic properties vary from metallic to semiconducting with diameter and chirality.

Since 2004, a sixth form of carbon has been defined that can be considered as a single layer of graphite called "graphene". The material has been produced by mechanical exfoliation of graphite (Geim \& Novoselov 2007). Other methods, developed to produce graphene, are $\mathrm{SiC}$ and hydrazine reduction, or chemical vapor deposition. One can consider graphene as an infinite or very large PAH partly hydrogen deficient. The largest molecule chemically synthesized in the laboratory is $\mathrm{C}_{222} \mathrm{H}_{42}$ with a mass of around $2700 \mathrm{u}(\mathrm{Wu}$ et al. 2007). In gasphase condensation processes, even larger molecules can be produced, however, to make them visible with analytical methods is rather difficult.

The very large graphene species have to be stabilized either by a substrate or by a scaffold. Graphene can be transformed into a hydrogenated form (graphane) by exposing the sheets to a cold hydrogen plasma (Savchenko 2009; Elias et al. 2009). The material transforms from a highly conductive material into an insulator. This process is reversible and can be reversed by annealing in argon. Density-functionalbased tight-binding level calculations have shown that the HOMO-LUMO gaps of graphene nanoflakes vary from zero to gap energies typical for insulators (Kuc et al. 2010).

\section{Structure of gas-phase condensed carbon nano-particles}

It is generally assumed that the major fraction of the primary cosmic carbonaceous material is formed as nanometer-sized particles via gas-phase condensation in circumstellar envelopes of carbon-rich asymptotic giant branch (AGB) stars (Henning \& Salama 1998). In order to understand the cosmic condensation of carbonaceous grains, gas-phase condensations of carbonaceous grains have to be simulated in the laboratory. Dedicated analytical methods such as high-resolution transmission electron microscopy (HRTEM) and UV/Vis/IR spectroscopy help to understand the structure and composition of condensed carbon grains. Here, we present a brief overview on the structural characteristics of gas-phase condensed nano-particles produced from carbon and hydrogen.

The structural complexity of pure carbon or carbon mixed with hydrogen can be huge in solid carbon. Differently hybridized carbon $\left(\mathrm{sp}, \mathrm{sp}^{2}\right.$, and $\left.\mathrm{sp}^{3}\right)$ and mixed hybridization states may occur in different ratios in one grain. The structure of particulate carbon materials can be characterized on different length scales describing the short-, medium-, and long-range order of the material. The longrange order describes the size and shape of the primary particles and their agglomeration state. The medium-range order characterizes the arrangement, shape and size of the structural subunits and can be derived by HRTEM. So-called basic 


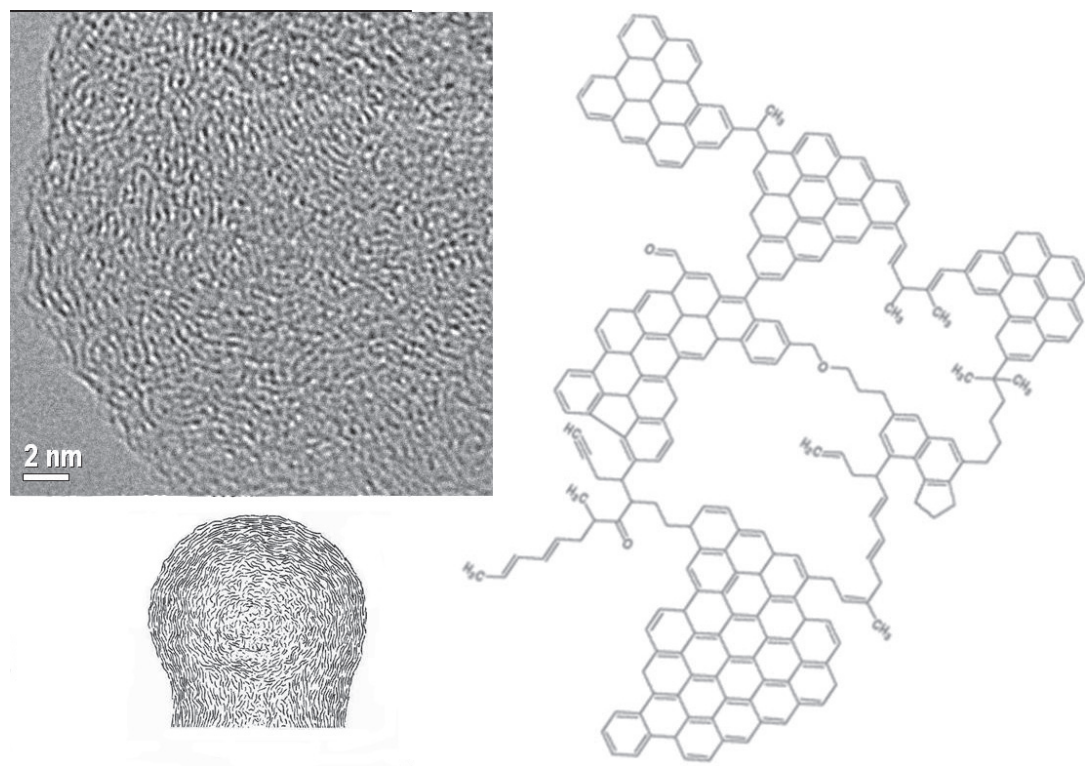

Fig. 1. HRTEM image of a gas-phase condensed particle having a paracrystalline structure. The chemical formula (right) shows one possible amorphous carbon structure which can be considered as a paracrystalline graphene layers.

structural units (BSUs) consisting of small subnanometer- or nanometer-sized graphitic crystallites which can be randomly or concentrically arranged in a grain have been already discussed in the sixties (Heidenreich et al. 1968). However, plenty of HRTEM studies of condensed particles show the presence of individual graphene layers that are concentrically arranged. This structure can be described by the more recent paracrystalline model of soot grains (Hess \& Herd 1993) which can be characterized by a layer structure of perturbed graphene layers. At present, this concept is the most widely accepted microstructural model for soot.

The hybridization state of the carbon, the nature of the bonds between carbon atoms as well as the incorporation of hydrogen or hetero atoms in the carbon grains can be specified by the short-range order of the material. Analytical methods like electron energy loss, IR, or nuclear magnetic resonance spectroscopy have to be applied to obtain the information on the short range order (Schnaiter et al. 1998; Jäger et al. 1999). The short- and the medium-range order of carbon grains can be well described by the terms "amorphous carbon" (AC) or "hydrogenated amorphous carbon" (HAC) (see Fig. 1). HACs are frequently used to describe the carbonaceous dust component in astrophysical studies (Jones et al. 1990; Duley 1994; Pendleton \& Allamandola 2002; Dartois et al. 2010). Both terms, "HACs" and $\mathrm{ACs}$, are misleading since they describe groups of carbonaceous materials with different $\mathrm{C} / \mathrm{H}$ ratios, varying ratios of $\mathrm{sp}^{3}, \mathrm{sp}^{2}$, and $\mathrm{sp}$ hybridized carbon, and gap 
energies between 0.5 and about $4.0 \mathrm{eV}$. They represent three-dimensional structures applicable for the characterization of graphitic or paracrystalline structural units in grains. In $\mathrm{AC}$ and $\mathrm{HAC}$, the carbonaceous structure can be characterized by aromatic islands of different sizes, which are linked by aliphatic structures including $\mathrm{sp}, \mathrm{sp}^{2}$, and $\mathrm{sp}^{3}$ carbon atoms. However, there is no clear discrimination between $\mathrm{AC}$ and $\mathrm{HAC}$ on the basis of the sizes of aromatic and aliphatic units and on their $\mathrm{H} / \mathrm{C}$ ratios. In astrophysical studies, the term HAC is preferentially used for carbon materials with very small aromatic units (2-8 rings Jones et al. 1990). Oxygen in the form of ether bridges (-C-O-C-) or carbonyl groups $(-\mathrm{C}=\mathrm{O})$ may also be included. However, the amount of oxygen incorporated into a solid hydrocarbon material present in the diffuse interstellar medium is assumed to be low (Pendleton \& Allamandola 2002). According to Robertson (1986), the aromatic as well as the aliphatic structures in HACs tend to cluster. Another acronym for similar materials were "quenched carbonaceous composites" (Sakata et al. 1993; Wada et al. 1999). The application of natural coals as cosmic dust analogs has been suggested by Papoular et al. (1993).

The spectral properties of HACs in the UV/Vis are dominated by electronic transitions. Whereas the $\sigma-\sigma^{*}$ transitions are located in the far $\mathrm{UV}$, the $\pi-\pi *$ transitions occur at wavelengths between 190 and $270 \mathrm{~nm}$. Robertson \& O' Reilly (1987) claimed that the size of the aromatic units determine the gap energy and the position of the $\pi-\pi^{*}$ transitions. The gap energy $E_{g}$ can be derived from the optical spectra by means of the Tauc relation $\sqrt{\epsilon_{2} E}=B\left(E-E_{g}\right.$ ) (Tauc et al. 1966). According to the relation $E_{g}=2 \beta M^{-1 / 2}$ derived by Robertson \& O'Reilly (1987), where $\beta$ represents a quantum chemically estimated overlapping energy between two adjacent $\mathrm{p}_{z}$ orbitals that has an empirical value of $2.9 \mathrm{eV}$, the optical gap energy of soot is related to the number $M$ of condensed rings in the graphene layers. However, nowadays it is widely accepted that bent graphene layers can be incorporated into particles. For mixed hybridization states, the electronic transitions are shifted to higher energies proven by using sophisticated spectral measurements such as electron energy loss or near edge X-ray absorption spectroscopy (Ajayan et al. 1993; Ravagnan et al. 2006; Jäger et al. 2010).

In the IR range, the functional groups included in the HAC structure can cause vibrational bands in absorption or emission. In addition, the sizes of aromatic subunits also influence the IR spectral properties due to continuous absorptions by free charge carriers. In particular, the sizes of aromatic subunits affect the conductivity of the material which has a strong influence on the absorptivity in the range between $100 \mu \mathrm{m}$ and $1 \mathrm{~mm}$.

A special group of gas-phase condensed grains are onion-like or fullerene-like grains including nested buckyonions. Faceted polyhedral onions and onions with not perfectly closed shells are formed during the condensation of carbon vapor at high temperature, for example, in the arc discharge. Completely spherical onions are non-equilibrium structures and self-assemble only under irradiation of graphitic or amorphous carbon precursors (Ugarte 1992; Banhart 1999). Nowadays, defective carbon onions were discussed to be possible carriers of different observational bands such as the $217 \mathrm{~nm}$ bump (Tomita et al. 2002; Chhowalla et al. 2003). 


\section{Soot formation}

\subsection{Soot formation in astrophysical environments}

Carbon grain condensation in astrophysical environments has been rarely discussed in the astrophysical literature. Gail \& Sedlmayr (1985) have applied classical nucleation theory to study the carbon grain formation in stationary, spherical expanding winds. They calculated condensation temperatures of dust between 1260 and $1320 \mathrm{~K}$ for optically thin and between 1350 and $1430 \mathrm{~K}$ for optically thick shells. The final grain radii were found to be small but very close to the theoretically possible maximum if all condensable material is included in grains. Lodders \& Fegley (1999) modeled dust condensation in carbon-rich AGB stars using thermodynamic equilibrium calculations and found that the condensation temperature of graphite is pressure independent but sensitive to the $\mathrm{C} / \mathrm{O}$ ratio in the circumstellar shells.

Detailed kinetic models for the formation of PAHs from acetylene in AGB stars have been presented by Frenklach \& Feigelson (1989), Cherchneff et al. (1992), Allain et al. (1997), Cherchneff (2010). The authors determined a narrow temperature range of 900 to $1100 \mathrm{~K}$ for the formation of PAHs in circumstellar environments strongly dependent on the basic set of conditions such as mass loss, wind velocity, and gas density. Frenklach \& Feigelson (1989) found a yield of 1.3\% with respect to the amount of carbon initially present in acetylene for the most favorable case (low effective temperature of $1500 \mathrm{~K}$, a mass loss rate as high as $10^{-4} M_{\odot}$ per year and a wind velocity of $10^{-2} \mathrm{~km} \mathrm{~s}^{-1}$ ). Cherchneff et al. (1992) obtained a maximum yield of $5 \times 10^{-5}$. Cherchneff \& Cau (1999) reconsidered the modeling of the PAH and carbon dust formation in carbon-rich AGB stars and applied a physico-chemical model, which describes the periodically shocked gas in the circumstellar shells close to the photosphere of the stars. The authors claimed that the formation of the first aromatic ring begins at $1.4 \mathrm{R}_{*}$ and, at a radius of $1.7 \mathrm{R}_{*}$ corresponding to a temperature of around $1700 \mathrm{~K}$, the conversion of single rings to PAHs begins. The resulting temperature is much higher than the temperature window calculated in previous studies. Cau (2002) investigated the contribution of periodic shocks to the formation of PAHs and their dimers in the inner atmosphere of IRC +10216 . He has shown that the amount of PAHs and dimers produced is not enough to explain the formation of carbon dust in the atmosphere of the star, but it can account for the formation of the small disordered cores of the kind observed in presolar grains. These cores might then grow larger through direct chemical reactions with acetylene. The yield of PAHs and dimers obtained was lower than that found by Frenklach \& Feigelson (1989).

\subsection{Soot formation in terrestrial combustion processes}

Most experimental data on the kinetics of soot formation were obtained in flames and shock waves. A rough picture of soot formation in flames has been provided by Richter \& Howard (2000) who distinguish three main steps of the soot formation. The process starts with the formation of the molecular precursors of soot which 
are supposed to be big PAHs of molecular weights larger than $300 \mathrm{u}$. The growth process from small molecules such as benzene to larger PAHs comprises both the addition of $\mathrm{C}_{2}, \mathrm{C}_{3}$ or other small units to $\mathrm{PAH}$ radicals and reactions among the growing aromatic species.

The second part of the process is called particle inception. In this process, the conversion from molecular to particulate systems happens. The third part of the soot formation is the particle growth process which can be again sub-divided into the growth of particles by addition of gas-phase molecules, such as PAHs, and coagulation via reactive particle-öparticle collisions, a process which significantly increases particles sizes. Whereas the first and third step is rather well understood the soot nucleation or particle inception is not yet understood. The first and the second step of soot formation are the most interesting ones for astrophysics.

However, one has to keep in mind that soot formation pathways in flames are more complicated than in the more isothermal laser pyrolysis or laser ablation processes. Within a flame the temperature is rising from the bottom to the top and from the outer edge to the middle of the flame resulting in different formation pathways to soot particles. The application of gas-phase condensation methods which provides higher homogeneity in the temperature distribution are necessary to obtain more general information on carbon grain formation in such processes.

In our laboratory, we have employed gas-phase condensation experiments such as laser pyrolysis and laser ablation in quenching gas atmospheres to condense carbonaceous particles within two different temperature regimes, a high- (HT) and a low-temperature (LT) condensation regime. More experimental details on the techniques can be found in Jäger et al. (2008) and (2009). In both cases, the gas-phase condensation is comparable to the grain formation processes suggested to be relevant in astrophysical environments.

The study of the formation pathways has been performed by analytical characterization of the complete carbonaceous condensate, including high-resolution transmission electron microscopy (HRTEM), chromatographic methods, and mass spectroscopy assuming that some fraction of the precursor molecules and intermediate products are preserved in the final condensate due to an efficient quenching of the condensed carbon materials after the formation. In LT condensation processes at temperatures lower than $1700 \mathrm{~K}$, either mixtures of insoluble grains and PAHs or exclusively PAHs are formed. The typical internal structure of the particles can be described by a concentrical arrangement of rather plane graphene layers around a kind of disordered core (see Fig. 2). Matrix-assisted laser desorption and ionization combined with time-of-flight (MALDI-TOF) mass spectrometer studies have demonstrated that PAHs up to masses of $3000 \mathrm{u}$ and even higher are formed during the condensation process (see Fig. 3). All sizes of PAHs are formed in such condensations but no saturated hydrocarbon molecules could be identified. LT condensation favors a formation process with PAHs as precursors and particle-forming elements, which condense on the surface of carbonaceous seeds via physi- and chemisorption. As a result, large carbonaceous grains are formed revealing well developed planar or only slightly bent graphene layers in their interior. Since larger PAHs have a lower volatility compared to the smaller ones, a 


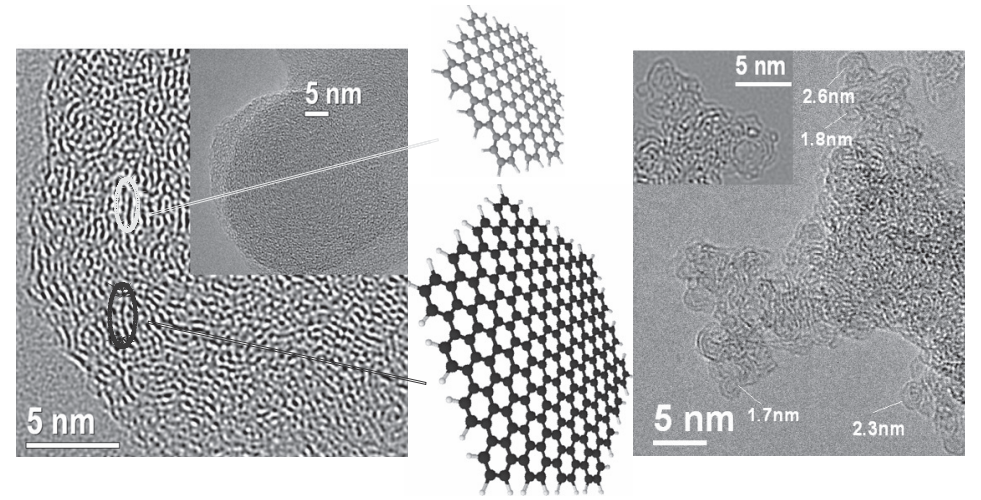

Fig. 2. HRTEM micrograph of a grain formed in a LT (left) condensation process. The average length of graphene layers is around $2 \mathrm{~nm}$ comparable to PAHs with masses of around $1300 \mathrm{u}$ (smaller PAH molecule). The largest layers have a size of $3 \mathrm{~nm}$ which corresponds to PAHs of about $2700 \mathrm{u}$ (large molecule). The HT condensed grains in the right panel are built of fullerene fragments or bucky onions with nested fullerene cages.

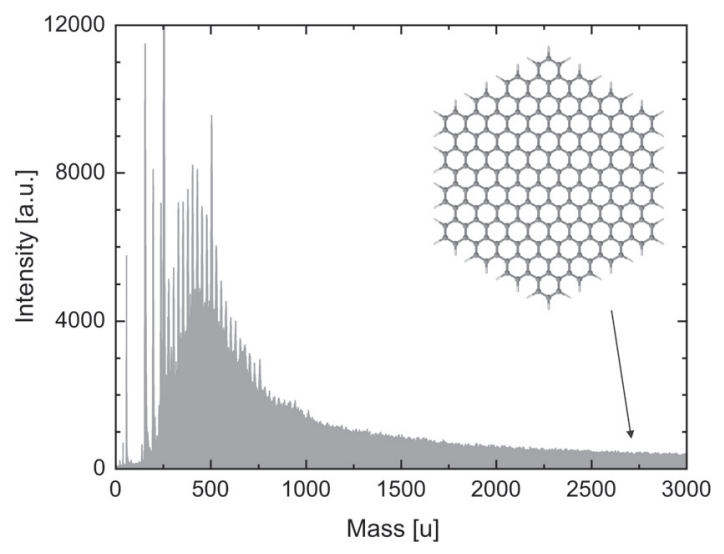

Fig. 3. MALDI TOF spectrum of a LT soot sample showing PAHs with masses up to $3000 \mathrm{u}$. To exemplify a PAH molecule with comparable mass, a molecule comprising 91 condensed rings is presented.

preferred accumulation of large molecules during the surface growth process can be observed. The accumulation of the large molecules on the surfaces of the seeds can be confirmed by a detailed analysis of the HRTEM micrographs (see Fig. 2) revealing a mean length of the graphene sheets inside the particles of around $2 \mathrm{~nm}$ corresponding to PAHs with masses of around $1300 \mathrm{u}$. The longest graphene layers determined in the soot particles have extensions of about $3 \mathrm{~nm}$ corresponding to the highest-mass PAHs detected with MALDI-TOF measurements (Jäger et al. 2009). 
The generation of fullerenes and fragments of them in the HT condensation process at temperatures higher than $3500 \mathrm{~K}$ could be verified by using electron microscopy which can be shown in Figure 2. The presence of symmetric and elongated fullerene molecules with different numbers of carbon atoms is clearly visible in the HRTEM micrographs (Jäger et al. 2009). Fullerene fragments, fullerenes and buckyonions serve as building blocks for the very small carbon grains formed in such HT regimes. MALDI-TOF studies of the condensate have shown that no PAHs are formed in this process pointing to a completely different soot formation pathway via polyyne chains, fullerenes and fullerene snatches. The formation pathway is found to be initiated by long and branched carbon macromolecules suggested to be the precursors for cyclic structures (Irle et al. 2003). The existence of polyyne structures in the condensate could be verified by in situ IR spectroscopy that shows the typical IR stretching bands of triply bound $\equiv \mathrm{CH}$ and $\mathrm{C} \equiv \mathrm{C}$ (Jäger et al. 2008).

\subsection{The nucleation process}

The controversial part of the soot formation even in the combustion community is the so-called inception, the formation of the first stable nuclei, i.e. the soot precursor particles.

Most of the combustion models favor the influence of PAHs and the formation of PAH clusters as soot nuclei (Dobbins et al. 1998; Richter \& Howard 2000; Mansurov 1998). Chen \& Dobbins (2000) considered large PAHs and PAH clusters with layer structure as nucleation seeds. Schuetz \& Frenklach (2000) discussed the dimerization of PAHs whereas Homann (1998) prefers the theory of PAH oligomerization resulting in the formation of so-called aromers. In $\mathrm{PAH}$ dominated soot formation processes, PAH radicals formed by hydrogen abstraction are discussed to be the driving force for the cluster formation (Richter et al. 2004). Reactions of $\mathrm{PAH}$ radicals with $\mathrm{PAH}$ and between $\mathrm{PAH}$ radicals were found to be the dominant pathway to soot nuclei. In flames, the transition from gaseous to solid particles was assumed to depend on the temperature and pressure. Particles heavier than $2000 \mathrm{u}$ and diameters of around $1.5 \mathrm{~nm}$ are considered as first solids (Lafleur et al. 1996; Harris \& Weiner 1988; McKinnon \& Howard 1992).

The formation of larger, three-dimensional species with amorphous carbon structure has been proposed by D'Alessio et al. (1998) and Minutolo et al. (1999). The role of fullerenes as soot nuclei (Zang et al. 1990) and the parallel growth of fullerenes and large PAHs in flames (Grieco et al. 2000; Lafleur et al. 1996) have been favored by other groups.

Generally, the exact transition range from molecules to solid grains is different for different materials and varies between clusters of 100 and 1000 atoms. A nice example for very small solid carbonaceous grains are the meteoritic nanodiamonds with a medium size of $1.3 \mathrm{~nm}$ corresponding to number of around 150 atoms. This points to a high stability of these "clusters" consisting of $\mathrm{sp}^{3}$ hybridized carbon exclusively. Transitional objects between molecules and solids in HT condensates have been observed by using HRTEM. Their structures are similar to the carbon 

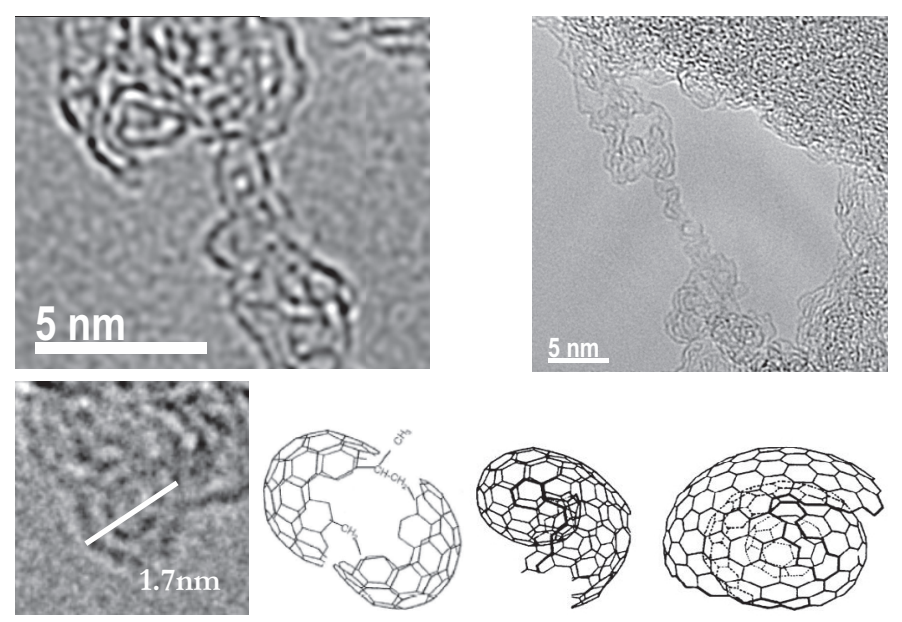

Fig. 4. Small transitional objects between molecules and solid soot grains observed in HT condensation experiments.

cluster with spiral structures (Haberland 1994) which are supposed to be intermediates of the soot formation in condensation processes with high initial carbon atom densities. Three-dimensional fragments of fullerenes, oligomers of fullerenes or fragments and small nested buckyonions have been identified in this condensate (Jäger et al. 2009).

\section{Conclusion and outlook}

Gas-phase condensation studies to uncover the formation process of carbon grains at different condensation conditions are necessary to understand the structural and spectral properties of nanometer-sized carbon grains with respect to an application to cosmic carbon. So far, these studies succeeded in the production and structural characterization of a big variety of carbon structures and in the tuning of special structural and spectral properties of the material.

A first basic understanding of the grain formation process in gas-phase condensations was reached. Laboratory studies have shown that in flames as well as in condensation processes with isothermal conditions, such as laser ablation or pyrolysis, multiple soot formation processes occur. In isothermal condensations, a high- and a low-temperature soot formation process have been identified in which either small fullerene-like carbon grains are produced via carbon chains, fullerenes and fullerene fragments, or soot grains with plane graphene layers are formed via PAHs, respectively. However, we still need more insight into the complicate nucleation process of soot grains, in particular in PAH dominated formation processes. Further laboratory experiments to study the inception or nucleation step have to be developed by coupling selective production methods with sophisticated 
analytical tools (Biennier et al. 2010). In high-temperature condensation processes, three-dimensional fragments of fullerenes and small nested buckyonions have been identified as transitional objects between molecules and solids.

\section{References}

Ajayan, P., Iijima, S., \& Ichihashi, T., 1993, Phys. Rev. B, 47, 6859

Allain, T., Sedlmayr, E., \& Leach, S., 1997, A\&A, 323, 163

Banhart, F., 1999, Rep. Prog. Phys., 62, 1181

Biennier, L., Sabbah, H., Chandrasekaran, V., et al., 2011, this volume

Cau, P., 2002, A\&A, 392, 203

Chen, H.X., \& Dobbins, R., 2000, Combust. Sci. Tech., 159, 109

Cherchneff, I., Barker, J., \& Tielens, A., 1992, ApJ, 401, 269

Cherchneff, I., \& Cau, P., 1999, in ed. T. Le Betre, A. Lèbre, and C. Waelkens, Asymptotic Giant Branch Stars; Proceedings of the IAU Symposium 191 (San Francisco, ASP), 251

Cherchneff, I., 2011, this volume

Chhowalla, M., Wang, H., Sano, N., et al., 2003, Phys. Rev. Lett., 90, 155504

D’Alessio, A., D’Anna, A., Gambi, G., \& Minutolo, P., 1998, J. Aerosol. Sci., 29, 397

Dartois, E., \& Godard, M., 2011, this volume

Dobbins, R.A., Fletcher, R.A., \& Chang, H.-C., 1998, Comb. Flame, 115, 285

Duley, W., 1994, ApJ, 430, L133

Elias, D.C., Nair, R.R., Mohiuddin, T.M.G.J., et al., 2009, Science, 323, 610

Frenklach, M., \& Feigelson, E., 1989, ApJ, 341, 372

Gail, H.-P., \& Sedlmayr, E., 1985, A\&A, 148, 183

Geim, A.K., \& Novoselov, K.S., 2007, Nature Materials, 6, 183

Grieco, W.J., Howard, J.B., Rainey, L.C., \& Vander Sande, J.B., 2000, Carbon, 38, 597

Haberland, H., 1994, Clusters of Atoms and Molecules (Springer Verlag, Heidelberg)

Haddon, R.C., 1993, in ed. H. Kroto and D. Walton, The fullerenes (Cambridge University Press, Cambridge)

Harris, P.J.F., 2009, Carbon Nanotube Science (Cambridge University Press)

Harris, S.J., \& Weiner, A.M., 1988, Proc. Combust. Inst., 22, 333

Heidenreich, R., Hess, W., \& Ban, L., 1968, J. Appl. Cryst., 1, 1

Henning, T., \& Salama, F., 1998, Science, 282, 2204

Hess, W., \& Herd, C., 1993, in ed. J. Donnet, R. Bansal, and M. Wang, Carbon Black (Marcel Dekker, Inc., New York), 89

Homann, K.-H., 1998, Angew. Chem., 110, 2572

Iijima, S., 1991, Nature, 354, 56

Irle, S., Zheng, G., Elstner, M., \& Morokuma, K., 2003, Nano Lett., 3, 1657

Jäger, C., Henning, T., Spillecke, N., \& Schlögl, R., 1999, J. Non-Cryst. Solids, 258, 161

Jäger, C., Huisken, F., Mutschke, H., Llamas Jansa, I., \& Henning, T., 2009, ApJ, 696, 706

Jäger, C., Kovacevic, E., Mutschke, H., Henning, T., \& Huisken, F., 2010, in preparation 
Jäger, C., Mutschke, H., Henning, T., \& Huisken, F., 2008, ApJ, 689, 249

Jones, A.P., Duley, W.W., \& Williams, D.A., 1990, QJRAS, 31, 567

Krätschmer, W., Lamb, L.D., Fostiropoulos, K., \& Huffman, D., 1990, Nature, 347, 354

Kroto, H., Heath, J., O'Brien, S., Curl, R., \& Smalley, R., 1985, Nature, 318, 162

Kuc, A., Heine, T., \& Seifert, G., 2010, Phys. Rev. B, 81, 085430

Lafleur, A.L., Taghizadeh, K., \& Howard, J.B. e. a., 1996, J. Amer. Soc. Mass Spectrom., 7,276

Lodders, K., \& Fegley, B., J., 1999, in ed. T. Le Bertre, A. Lebre, and C. Waelkens, Asymptotic Giant Branch Stars; Proceedings of the IAU Symposium 191, SAO/NASA Astrophysics Data System, 279

Mansurov, Z.A., 1998, Combust Flame, 115, 285

McKinnon, J.T., \& Howard, J.B., 1992, Proc. Combust. Inst., 24, 965

Minutolo, P., Gambi, G., D’Alessio, A., \& Carlucci, S., 1999, Atmospheric Environment, 33,2725

Papoular, R., Breton, J., Gensterblum, G., et al., 1993, A\&A, 270, L5

Pendleton, Y.J., \& Allamandola, L.J., 2002, ApJS, 138, 75

Ravagnan, L., Bongiorno, G., Bandiera, D., et al., 2006, Carbon, 44, 1518

Ravagnan, L., Siviero, F., \& Lenardi, C. e. a., 2002, Phys. Rev. Lett., 89, 285506

Richter, H., Granata, S., Green, W.H., \& Howard, J.B., 2004, Proc. Combust. Inst., 30, 1397

Richter, H., \& Howard, J.B., 2000, Prog. Energy Combust. Sci., 26, 565

Robertson, J., 1986, Adv. Phys., 35, 317

Robertson, J., \& O'Reilly, E., 1987, Phys. Rev. B, 35, 2946

Sakata, A., Wada, S., Okutsu, Y., Shintani, H., \& Nakada, Y., 1993, Nature, 301, 493

Savchenko, A., 2009, Science, 323, 589

Schnaiter, M., Mutschke, H., Dorschner, J., Henning, T., \& Salama, F., 1998, ApJ, 498, 486

Schuetz, A.C., \& Frenklach, M., 2000, Combust. Sci. Tech., 159, 109

Tauc, J., Grigorovici, R., \& Vancu, A., 1966, Phys. Status Solid, 15, 627

Tomita, S., Fujii, M., \& Hayashi, S., 2002, Phys. Rev. B, 66, 245424

Ugarte, D., 1992, Nature, 359, 707

Wada, S., Kaito, C., Kimura, S., Ono, H., \& Tokunaga, A.T., 1999, A\&A, 345, 259

Wu, J., Pisula, W., \& Müllen, K., 2007, Chem. Rev., 107, 718

Zang, Q.L., O'Brien, S.C., \& Heath, J.R. e. a., 1990, J. Phys. Chem., 90, 525 